\title{
A Note on the Exponents of Lyapunov for a System with Unknown Dynamic
}

\author{
Pedro Pablo Cárdenas Alzate ${ }^{1 *}$, José Gerardo Cardona Toro² and Luz María Rojas Duque ${ }^{3}$ \\ 'Departamento de Matemáticas and Research Group GEDNOL, Universidad Tecnológica de Pereira, \\ Pereira, Colombia; ppablo@utp.edu.co \\ 2 Departamento de Matemáticas, Universidad Tecnológica de Pereira, Pereira, Colombia; gerardo7@utp.edu.co \\ ${ }^{3}$ Fundación Universitaria del Área Andina, Pereira, Colombia; Imrojas@areandina.edu.co
}

\begin{abstract}
Objective: To determine the exponents of Lyapunov for a system with unknown dynamics. Methods: The determination of chaos presence in any system have a great importance to right analysis, thus there are indicators that permit quantifying this phenomenon, one of them and perhaps more relevant are the Lyapunov exponents. That is why this paper presents a methodology to calculate these quantifiers from the system dynamically. (differentials equations). Findings: In many occasions, we know the system dynamics, but we do not have necessary instruments to measure all the present states, thus we need to reconstructthe phase space from the times series scalar that we have. If one wants to analyze the original dynamics of the dynamic system from the said series, the attractor in the multivariable space is necessary, which is known as reconstruction of the phase space, originally proposed by Ruelle. This technique converts a scalar series $x(t)$ in a vector $v$ of dimension $m$ (Embedding dimension) using a delay time. Application: As mentioned, in various physical situations there is no measurement of all the variables of the system's state, therefore, it is necessary to reconstruct the dynamics of the system from the available measurements. Assuming that only the voltage measurement of capacitor one (Vc1) is available from the Chua circuit.
\end{abstract}

Keywords: Chua Circuit, Embedment Dimension, Exponents of Lyapunov, Multivariable Dynamic System, Phase Space

\section{Introduction}

Dynamic systems can be described by a set of ordinary differential equations, which when going into detail in terms of their modeling increases their complexity, becoming non-linear. The analysis of linear dynamic systems in phase space has reduced behaviors (fixed points, periodic or divergent solutions); in terms of non-linear systems they contain a variety of scenarios, stationary or convergent solutions (fixed points), periodic solutions (limit cycles), quasi-periodic (attractor bull) or chaotic solutions (strange attractors).
The chaos has been widely studied by mathematicians and engineers since its discovery by Edward Lorenz in 1970s. The main characteristic of these systems is the extreme sensitivity to the initial conditions, that is, for nearby trajectories initially their distance in the state space diagram will increase exponentially, being quantified by the exponents of Lyapunov ${ }^{1}$.

If you want to determine the exponents of Lyapunov for a system with unknown dynamics, you must reconstruct the phase space that captures the original attractor of the system. The most familiar method for the reconstruction of phase space from a scalar time series is based 
on the determination of a delay time and an embedding dimension ${ }^{2}$.

\section{Estimation of the Lyapunov Exponents}

\subsection{Phase Space}

When observing a real multivariable dynamic system, it is normal that all it's variables cannot be measured simultaneously, in the worst case there is only one measurement (time series).

$$
\begin{aligned}
& \vec{v}(t)=\left[v_{1}(t) v_{2}(t) \ldots v_{n}(t)\right]^{T} \\
& \vec{v}(t)=[x(t) x(t+\tau) \ldots x(t+(m-1) \tau)]^{T} \in R^{m}
\end{aligned}
$$

where $T$ is the transpose.

The proper choice of delay time and embedment dimension is very important for the correct reconstruction of the attractor. There are methods to determine the delay time, such as the false neighboring neighbors. On the other hand, the dimension of embedding of a $\mathrm{n}$-dimensional system is obtained according to the formulation of Takens ${ }^{3}$.

$$
\mathrm{m}^{2} \mathrm{n}+1
$$

\subsection{Lyapunov Exponents}

For an $n$-dimensional system described by

$$
\mathrm{x}(\mathrm{t})=\mathrm{F}(\mathrm{x}, \mathrm{t})
$$

the $i$-th exponent of Lyapunov is defined $\lambda_{\mathrm{i}}$ depending on the variations of the trajectories from a starting point $\mathrm{x}_{0}$ in $\mathrm{t}_{0}$, these variations can be seen as the degeneration of the radius of a hyper sphere $\delta_{0}(\mathrm{t})$ in a hyper-ellipsoid of radio $\delta_{\mathrm{i}}(\mathrm{t})$

$$
\delta_{\mathrm{i}}(\mathrm{t})=\delta_{0} \mathrm{e}^{\lambda_{\mathrm{i}}\left(\mathrm{t}-\mathrm{t}_{0}\right)}
$$

The classic procedure for the estimation of the exponents of Lyapunov was proposed by Wolf in $1985^{4}$, which consists of evaluating the local divergences from the application of the tangent plane associated with the system of state equations on $n$ orthogonal vectors

$$
\left\{\delta_{\mathrm{ix}}(\mathrm{t}), \delta_{2 \mathrm{x}}(\mathrm{t}), \ldots, \delta_{\mathrm{nx}}(\mathrm{t})\right\}
$$

Initially defined by the identity matrix $I_{n}$ The variational expressions give rise to the tangent space, defined as:

$$
\dot{\phi}(\mathrm{x}, \mathrm{t})=\mathrm{J}(\mathrm{x}, \mathrm{t}) \phi(\mathrm{x}, \mathrm{t})
$$

Where $\mathbf{J}(\mathbf{x}, \mathbf{t})$ represents the Jacobian of $\mathbf{F}(\mathbf{x}, \mathbf{t})$. The system of equation of state together with the variational expressions are integrated from $\mathrm{x}_{0}$ with $\phi\left(\mathrm{x}_{0}\right)=\mathrm{I}_{\mathrm{n}}$ for a time $T$ and thus obtain the divergent vectors transformed by the application of the tangent plane, that is, for the first exponent

$$
\delta_{1 \mathrm{x}}^{(1)}=\phi(\mathrm{x}, \mathrm{t}) \mathrm{u}_{1}^{(0)}
$$

Where $\mathrm{u}_{1}^{(0)}=\delta_{1 \mathrm{x}}^{(1)} / \delta_{1 \mathrm{x}}^{(1)}$ and the superscript represents the current iteration of the calculation. By repeating this procedure of integration and normalization $K$ times the $i$-th exponent of Lyapunov can be written in the following way:

$$
\lambda_{i}=\lim _{K \rightarrow \infty} \frac{1}{K T} \sum_{k=1}^{K} \ln \delta_{i x}^{(k)}
$$

In each iteration made the direction of the vectors $\left\{\delta_{\text {ix }}(\mathrm{t}), \delta_{2 \mathrm{x}}(\mathrm{t}), \ldots, \delta_{\mathrm{nx}}(\mathrm{t})\right\}$ it must be aligned with the expansion direction of the system dynamics, for this reason the Gram-Schmidt method must be used, as shown below

$$
\begin{aligned}
& \mathrm{v}_{\mathrm{n}}^{(\mathrm{k})}=\delta_{\mathrm{nx}}^{(\mathrm{k})}-\left\langle\delta_{\mathrm{nx}}^{(\mathrm{k})}, \mathrm{u}_{1}^{(\mathrm{k})}\right\rangle \mathrm{u}_{1}^{(\mathrm{k})}-\ldots\left\langle\delta_{\mathrm{nx}}^{(\mathrm{k})}, \mathrm{u}_{\mathrm{n}-1}^{(\mathrm{k})}\right\rangle \mathrm{u}_{\mathrm{n}-1}^{(\mathrm{k})} \\
& \mathrm{u}_{\mathrm{n}}^{(\mathrm{k})}=\frac{\mathrm{v}_{\mathrm{n}}^{(\mathrm{k})}}{\mathrm{v}_{\mathrm{n}}^{(\mathrm{k})}}
\end{aligned}
$$

Once the exponents of Lyapunov have been calculated, the maximum number of them must be identified, since it is the one in charge of determining the presence of chaos or not in said dynamic system. For this, it is clear that if you have $\lambda_{\max }>0$ the initially close trajectories in the phase space will tend to separate, that is, the system presents a high sensitivity to the initial conditions.

\section{Application to Chua's Circuit}

Since its discovery in 1983, the Chua circuit has served as the basis for the study of the chaos in electronic systems ${ }^{5}$. This circuit is composed of two capacitors ( $\mathrm{C} 1$ and $\mathrm{C} 2$ ), an 
inductance $(\mathrm{L})$, a resistance $(\mathrm{R})$ and a non-linear resistance $(\mathrm{Rn})$, in Figure 1 the canonical circuit can be observed.

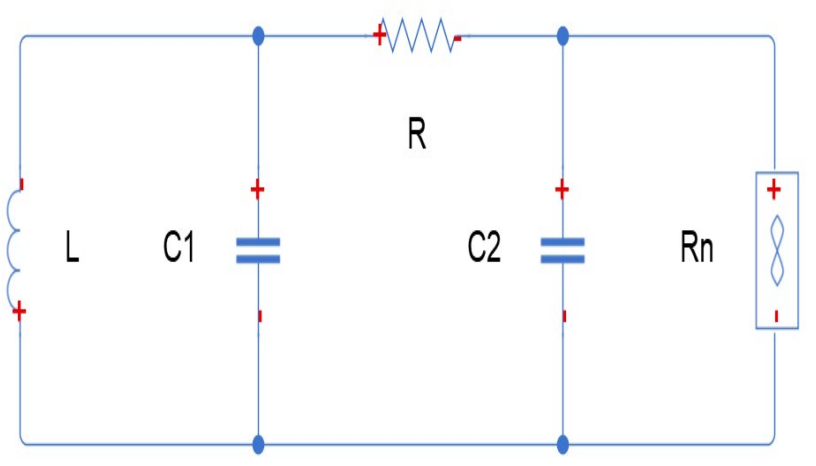

Figure 1. Chua's circuit.

Solving the circuit by state variables, the system is described by differential equations:

$$
\begin{aligned}
& \mathrm{x}_{1}=\frac{1}{\mathrm{C}_{1} \mathrm{R}}\left(\mathrm{x}_{2}-\mathrm{x}_{1}\right)-\mathrm{f}\left(\mathrm{x}_{1}\right) \\
& \mathrm{x}_{2}=\frac{1}{\mathrm{C}_{2} \mathrm{R}}\left(\mathrm{x}_{1}-\mathrm{x}_{2}\right)+\mathrm{x}_{3} \\
& \mathrm{x}_{2}=\frac{-1}{\mathrm{~L}} \mathrm{x}_{2}
\end{aligned}
$$

Where $\mathrm{x}_{1}=V_{C 1}, x_{2}=V_{C 2}, x_{3}=i_{L} f\left(x_{i}\right)=f\left(V_{C 1}\right)$ is the function that characterizes non-linear resistance, and is defined as follows?

$$
f\left(x_{1}\right)=m_{1} x_{1}+\frac{1}{2}\left(m_{0}-m_{1}\right)\left(\left|x_{1}+E\right|-\left|x_{1}-E\right|\right)
$$

Here, $E$ is a parameter dependent on the polarization voltage of the circuit, when implemented with OPAMs. The mathematical model of the Chua circuit can be normalized according to the proposal by Matsumoto ${ }^{5}$, for greater ease in the simulation

$$
\begin{aligned}
& x=\alpha(y-h(x)) \\
& y=x-y+z \\
& z=-\beta y
\end{aligned}
$$

$\alpha, \beta$ being system parameters with typical values are $\alpha=9$, $\beta=14.286$. Here, $\mathrm{h}(\mathrm{x})$ it is represented in the form:

$$
h(x)=m_{1} x+\frac{1}{2}\left(m_{0}-m_{1}\right)(|x+1|-|x-1|)
$$

The values of the constants $\mathrm{m}_{0}$ and $\mathrm{m}_{1}$ are assumed for this analysis with $-1 / 7$ and 2/7 respectively.

By integrating the system (9) using the Runge-Kutta45 algorithm, the time series is obtained (Figure 2).

Graphing the phase diagram between $\mathrm{Vc1}$ and $\mathrm{Vc} 2$ shows that the resulting attractor is a limit cycle.

By varying the control parameter $\alpha=9$ (Figure 3), a large change in the dynamics of the system is observed, becoming a chaotic system (strange attractor) as seen in the phase diagram of Figure 4.

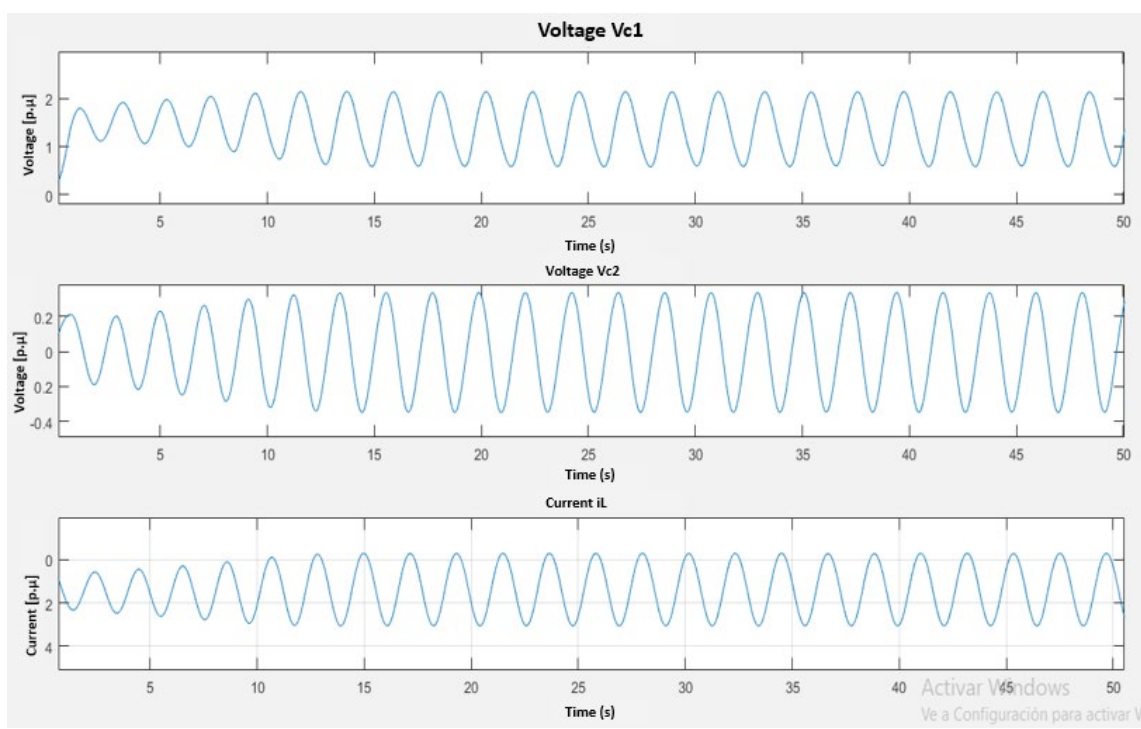

Figure 2. Time series $\mathrm{Vc1}, \mathrm{Vc} 2$ and iL with $\alpha=7.5$. 

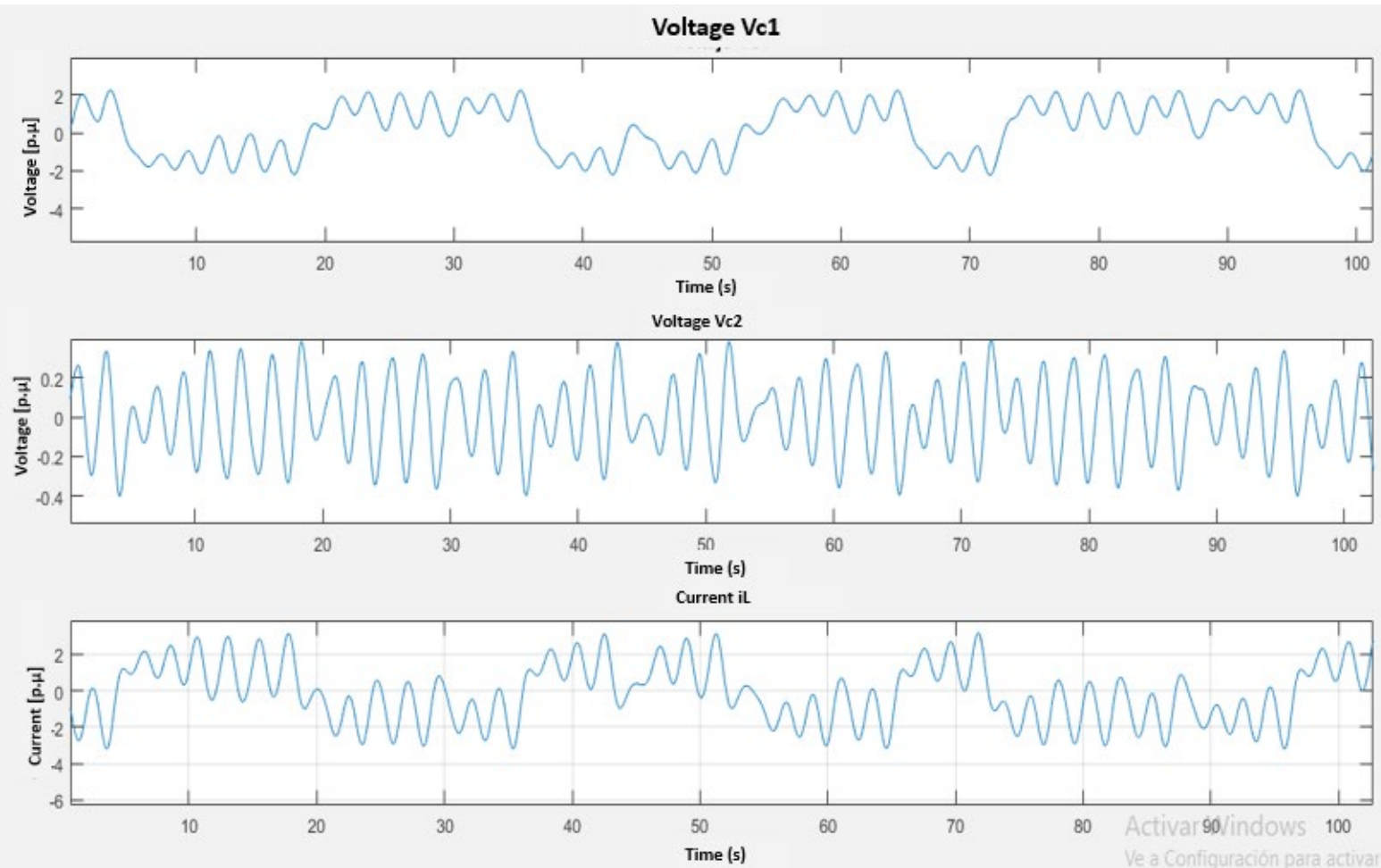

Figure 3. Time series with $\alpha=9$.

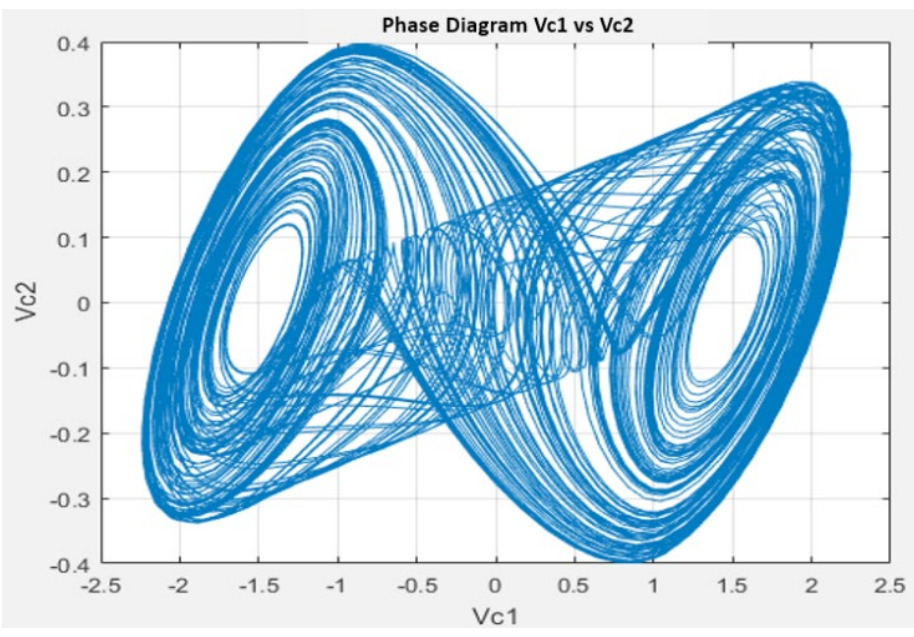

Figure 4. Strange attractor for $\alpha=9$.

\subsection{Reconstruction of the Phase Space of Chua's Circuit}

As mentioned, in various physical situations there is no measurement of all the variables of the system's state, therefore, it is necessary to reconstruct the dynamics of the system from the available measurements. Assuming that only the voltage measurement of capacitor one $(V c 1)$ is available from the Chua circuit, is (Figure 5):
Taking as dimension of embedment $\mathrm{m}=3$ and a delay time of $\tau=5 \mathrm{~s}$, the attractor of the system seen in Figure 6 is obtained.

\subsection{Determination of Lyapunov Exponents for the Chua Circuit}

For the calculation of the Lyapunov exponents ${ }^{6}$ of the Chua circuit, we start from the system (9), from which the Jacobian matrix is identified: 


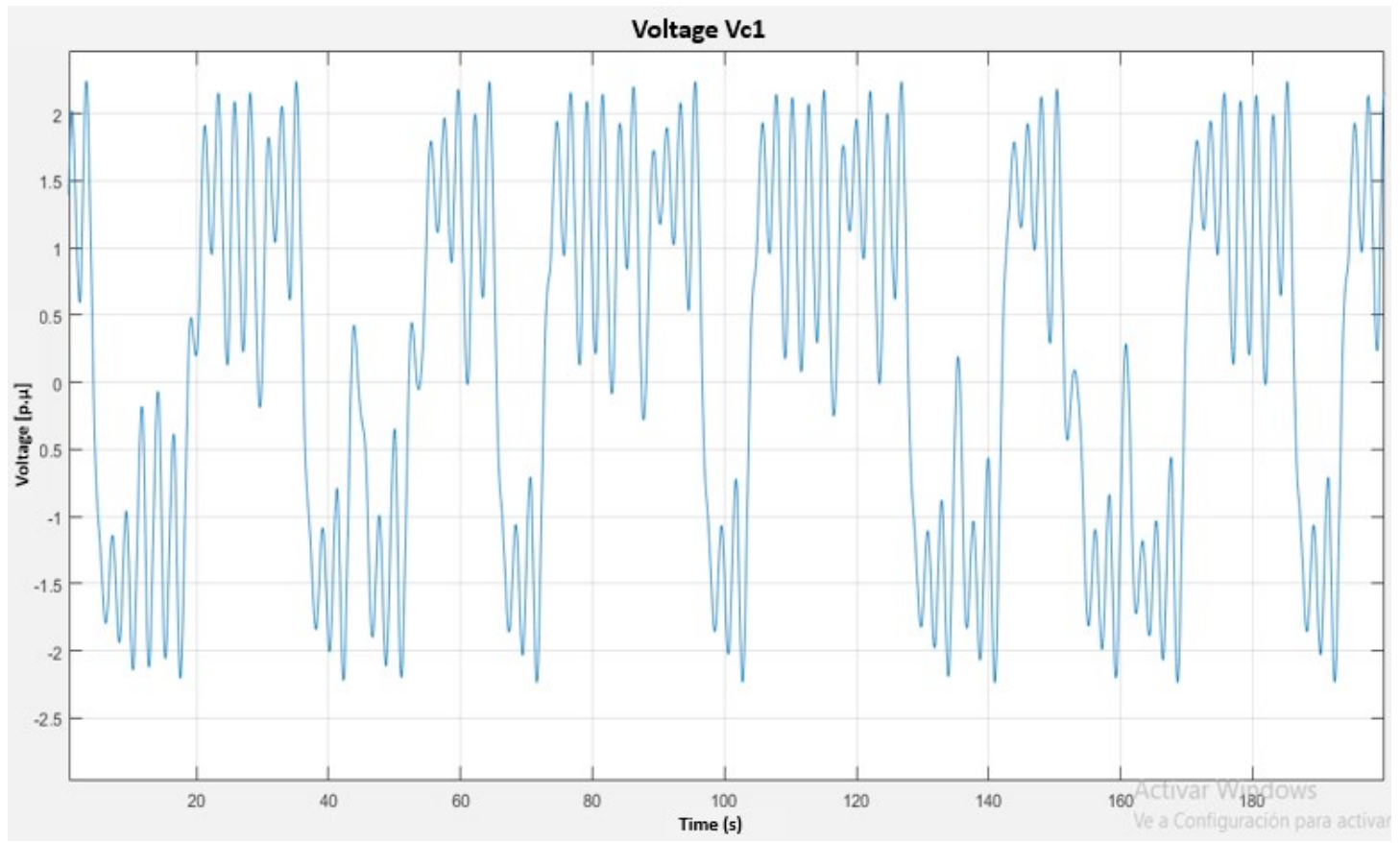

Figure 5. Measurement of $\mathrm{Vc1}$.
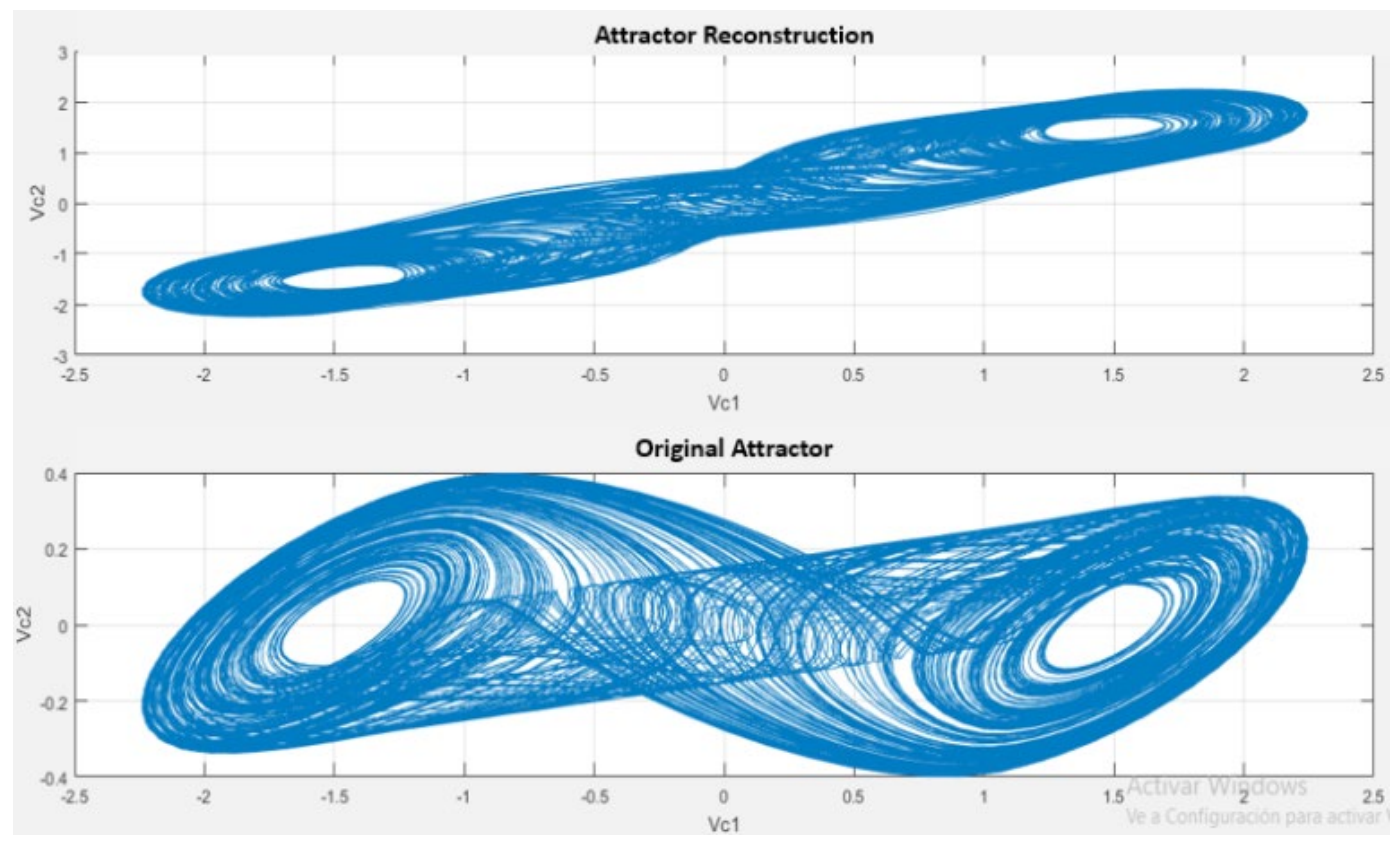

Figure 6. Comparison reconstructed vs. Original.

$$
\mathrm{J}(\mathrm{x}, \mathrm{t})=\left(\begin{array}{ccc}
\alpha \frac{\mathrm{dh}}{\mathrm{dx}} & \alpha & 0 \\
1 & -1 & 1 \\
0 & -\beta & 0
\end{array}\right)
$$

Which is the basis to start with the algorithm proposed by Wolf ${ }^{4}$, for the determination of the exponents of Lyapunov. The simulation (Figure 7) was performed with $\mathrm{T}=200, \alpha=9$ and initial conditions $[0.15264-0.02281$ $0.38127]$. 


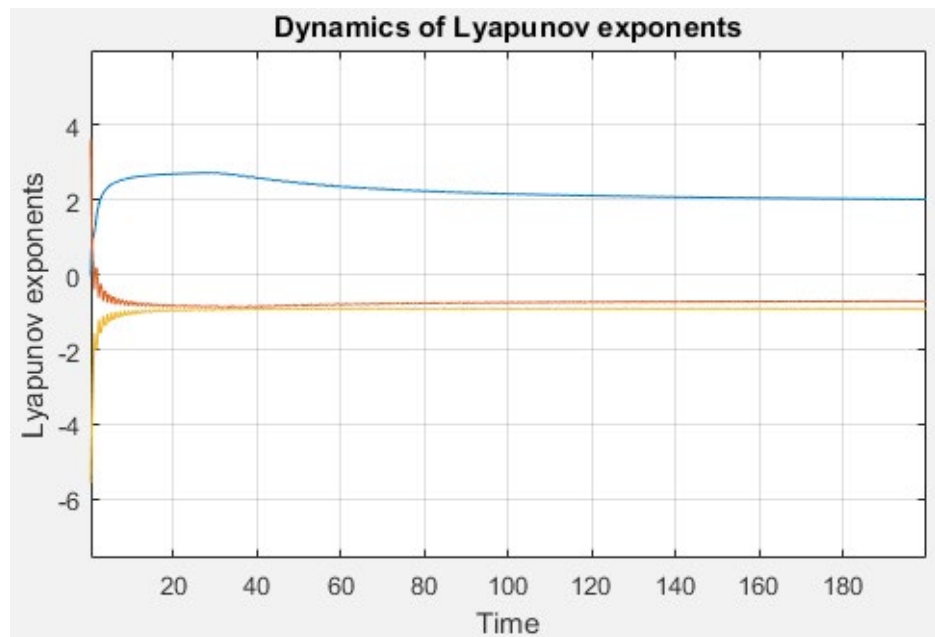

Figure 7. Spectrum of Lyapunov exponents for $\alpha=9$.

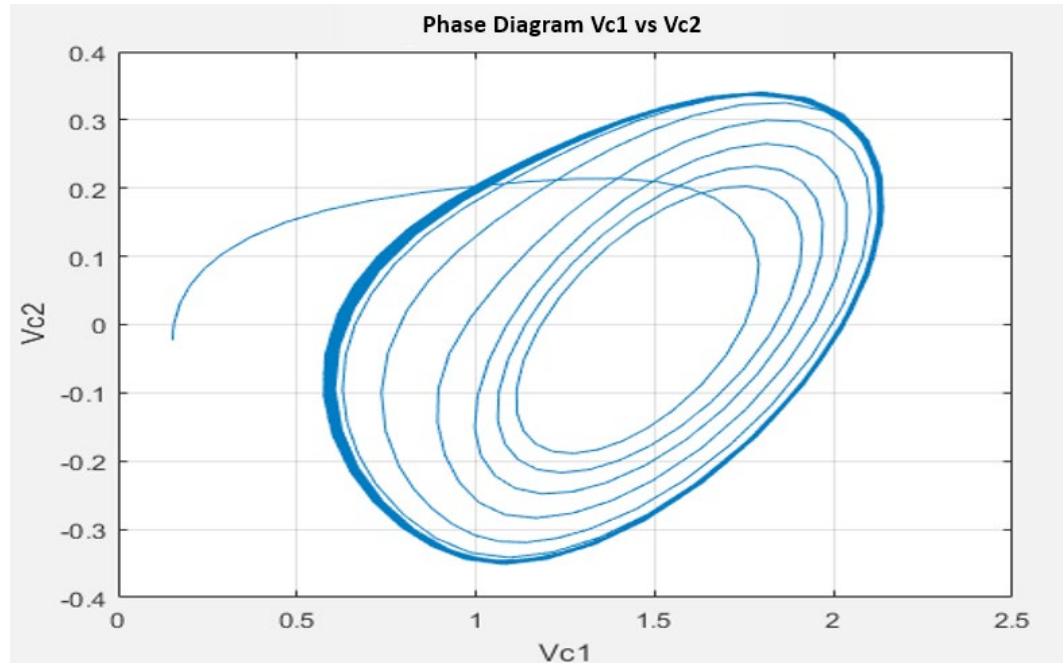

Figure 8. Limit cycle attractor $\alpha=7.5$.

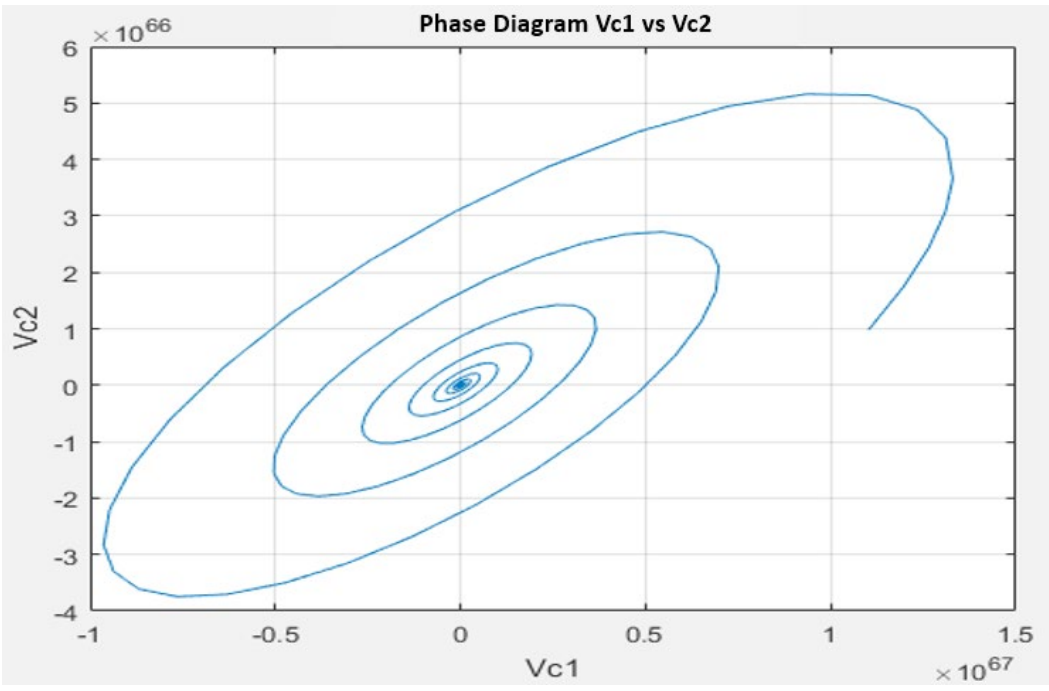

Figure 9. Divergence for $\alpha=10$. 
The values obtained are shown in Table 1. It is observed that the maximum exponent of Lyapunov $\lambda_{\max }=1.920399>0$, therefore the system will behave chaotic and its future states cannot be determined from the initial states. From the results of Figures 8-9, 4 a high dependence of the dynamics is observed with respect to the parameter $\alpha$.

Table 1. Exponents of Lyapunov for $\alpha=9$ which shows that the phase diagram is a strange attractor

\begin{tabular}{|l|l|l|l|}
\hline$\alpha$ & $\lambda_{1}$ & $\lambda_{2}$ & $\lambda_{3}$ \\
\hline 9 & 1.920399 & -0.686016 & -0.909888 \\
\hline
\end{tabular}

\section{Conclusions}

The characterization of the dynamics of any system is determined by exponents of Lyapunov, which, depending on their value, allow a qualitative analysis, establishing that dynamic is converging to a fixed point of the state space, or if it is periodic (limit cycle and bull) or simply if the future states cannot be determined from the initial states (strange chaotic-attractor system). Therefore, the constructive form of the circuit, for values between 7 and 11 there is a highly variable spectrum of the exponents of Lyapunov as it passes from one moment to operate stably under a limit cycle to be divergent. Finally, based on the knowledge of the dynamics of a system, a control law can be proposed to stabilize the system around the desired operating point that is why the phase space must be reconstructed when this dynamic is not known.

\section{Acknowledgements}

We would like to thank the referee for his valuable suggestions that improved the presentation of this paper and our gratitude to the Department of Mathematics of the Universidad Tecnológica de Pereira (Colombia) and the group GEDNOL.

\section{References}

1. Strogatz SH, Fox R. Nonlinear dynamics and chaos: With applications to physics, biology, chemistry and engineering. Physics Today. 1995; 48(3):48-93. Crossref

2. Eckmann JP, Ruelle D. Ergodic theory of chaos and strange attractors. Reviews of Modern Physics. 1985; 57:1-617. Crossref Crossref

3. Takens F. Detecting Strange Attractors in Turbulence. Dynamical Systems of Turbulence. 1981; p.366-81. Crossref

4. Wolf A, Swift J, Swinney H. Determining Lyapunov exponents from a time series. Physica D: Nonlinear Phenomena. 1985; 16 (3):285-317. Crossref

5. Elwakil A, Kennedy M. Generic realizations of Chua's circuit. International Journal of Bifurcation and Chaos. 2000; 10(8):1981-5. Crossref Crossref

6. Matsumoto T, Chua L, Komuro M. The double scroll. IEEE Transactions on Circuits and Systems CAS-32. 1985; (8):798-818. 